ABSTRACT OF

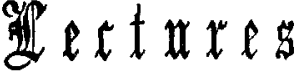

\author{
oN
}

\section{DISEASES OF THE GALL-BLADDER AND BILE-DUCTS.}

Delivered at the Royal College of Surgeons of England on March 8th, 10th, and 12th, 1897,

By A. W. MAYO ROBSON, F.R.C.S. EnG., HUNTERIAN PROFESSOR OF SURGERY AND PATHOLOGY, ROYAL COLLEGE OF SURGEONB OF ENGLAND.

\section{LECTURE II. ${ }^{1}$}

Delivered on March 10th.

Fistula._Fistulæ in connexion with the bile passages are hy no means uncommon, and their variety is considerable. They result from operation, or from disease, and in the latter case they are due to ulceration resulting from gall-stones or cancer. The fistulous channel may either be direct or indirect; in the former case being caused by an advancing nlcer setting up local peritonitis, and causing adhesion of the gall-bladder or bile-ducts to one of the neighbouring hollow viscera, or to the parietal peritoneum. The extension of the ulcer continuing, a communication is established with the contiguous channel or with the surface. In the latter case, (indirect) the perforation occurs first into an adjoining parenchymatous organ or into a localised abscess, and then into the nearest hollow viscus or on to the surface of the body at some part. A fistula may also arise from a local abscess forming outside the biliary passages around the primary focus of inflammation, and then bursting into the adjoining cavities, which are thus made to communicate. Although the establishment of a fistula is at times dangerous, and at others excessively annoying or uncomfortable, in many cases it forms one of nature's methods of cure in certain forms of disease of the gall-bladder or bile.ducts, and the surgeon in forming a permanent biliary fistula in otherwise incurable jaundice or in making an anastomosis between the bile passages and the intestine for the like purpose, is taking a leaf from nature's book. Many of the fistulæ are mere pathological curiosities, quite undiagnosable, and capable only of being discovered post mortem. Many must form and heal, leaving the patient cured, and thus never be discovered; for contrary to what one might suppose, fistulæ between the bile passages and other hollow viscera, in the majority of cases heal spontaneously, leaving only visceral adhesions; so that the fistula are comparatively rarely found post mortem. It would be of value if I could give statistics of the number of times that fistula follows an operation, but this I find is seldom mentioned by operators. I must, therefore, fall back on my own experience. My operations extend to 170 cases, 115 of which were cholecystotomies. I find that in 12 cases there were fistnlæ following, but as 5 occurred in my first 10 operations, since which time I have altered my method of procedure, it is fairer to say that 7 fistalæ occurred in 160 cases. Several of the fistulæ were inevitable, as the ducts were strictured; in others they were intentional, as in cancerous obstruction producing jaundice. Where the patients lived-i.e., where the obstruction was due to simple and not malignant disease, they were all cured by further operative means except my first patient, and she might be, but she says the small mucons fistula gives her so little trouble that it is not worth her loss of time to have it remedied.

Biliary cutaneous fistula.-Courvoisier's statistics gathered from reported cases would seem to prove that this is the commonest form of fistula. It may be pathological or postoperative.

Pathological surface fistule usually open at the umbilicus, the abscess following the course of the remains of the nmbilical vein, but they may form at any part of the abdominal wall, even near the pubes, or on the left side of the abdomen. Calculi of various sizes, from a single one,

1 Lecture I. appeared in The Lancet of May 29th, 1897. No. 3849 three inches in diameter, reported by Gutteridge, ${ }^{2}$ to multiple small faceted ones, the size of shotcorns, may be discharged in this way, leading to recovery and permanent cure, but until all the calculi are discharged the fistula is liable to remain open. In operating on these cases it is advisable to purify the fistulæ as far as possible and to scrape away all granulations before opening the peritoneal cavity to get at, and clear, the bile-ducts. By adopting these precautions I have had no untoward results in those cases on which I have operated.

Post-operative fistulce may be mucous or biliary.

Mucous fistule are occasionally seen after the operation of cholecystotomy, where the obstruction in 1 he cystic duct has not been overcome, or where that dact is the seat of stricture. In two cases of mucous fistula dependent on stricture of the cystic duct I removed the gall-bladder, effecting a complete and permanent cure. In another case, where a muco-purulent fistula had been discharging at the umbilicus for some months, I followed the channel up to the gall.blasder and found the cystic duct occlnded by calculi, which I removed, after which the fistula closed without difficulty. A mucous fistula, as a matter of fact, causes very little inconvenience, as only about $1 \mathrm{oz}$. of fluid is discharged daily; but if the opening be allowed to close the accumulation produces pain, and it is therefore necessary for a patient under these circumstances either to wear a small tube and a pad of absorbent wool or to submit to operation. The oneration of cholecystotomy will not be followed by fistula if the bile-ducts have been cleared, and if the opening in the gall-bladder be sutured to the aponeurosis and not to the skin. Since I modified the operation of cholecystotomy in this way, which I did in $\mathrm{my}$ eleventh operation, I have never known a fistula to follow when the bile-ducts have been cleared.

Biliary fistula following on operation is quite a different matter from mucous fistula, as, although in some cases it is compatible with good health, the inconvenience caused by thirty ounces of bile flowing from the fistula daily, produces so much discomfort that in all the cases which have come under my notice the patients have preferred to accept the risks of operation rather than to retain their disability. The treatment of biliary fistula should, where possible, be effected by removing the cause; but as in certain cases this is impracticable or impossible other means have to be considered. If the ducts be clear and the fistula be small the application of the actual cautery to the margin of the fistula will frequently result in its closure. That failing, the method I adopted in Case 116 may be followed, of opening the abdomen, detaching the gall-bladder, and suturing the opening. Or the less severe method may be tried first of dissecting the fistula with its peritoneal covering intact from the skin margin, afterwards donbling in the mucous edges, suturing them accurately, and over this applying one or two layers of buried sutures before bringing together the skin. Where, however, the ducts cannot be cleared and the gall-bladder is large enough to permit it. the operation of cholecystenterostomy may be performed. I believe I was the first to carry this operation out in a case of biliary fistula on Jan. 14th, 1888, and the patient is at the present time in excellent health, doing duty as a maternity nurse. I have since performed it on four occasions for fistula, each time with success, so that in such cases I feel I can recommend it as a safe and efficient method of treatment. If the fistula be dependent on gall-stones or fragments in the ducts the ducts may be syringed through daily with olive oil or with a 0.5 per cent. solution of sapo animalis, as recommended by Dr. Brockbank; or a solution of turpentine in ether $m$ iy be used. This is easily done by employing a small, flexible catheter, which is passed through the fistula as far as it will go without force. To the end of this a syringe is affixed and the medicament steadily syringed directly on to the obstruction, the syringing being repeated night and morning for a time.

Biliary intestinal fistulce, as might be expected from the contiguity of the gall-bladder to the duodenum and colon, are the most common, and as a rule they are due to ulceration produced by gall-stones. Usually the ulceration proceeds quietly and produces very few symptoms until the gall-stone sets up obstruction in its passage down the intestinal canal. If the fistula is between the gall-bladder and duodenum, "the most common variety," the whole length of the intestinal canal has to be traversed by the concretions; hence such cases are found to be more frequently
2 The LaNCET, June 8th, 1878, p. 851. 
assiociated with obstruction than when the fistula is bttween the gall-bladder and colon, for in the latter case the passage to the anus is accomplished without difficulty, though occasionally the concretions may lodge in the cæcum and cause trouble. Wrien a gall-stone is impacted in the common duct just before entering the duodenum ulceration and perforation of the duct are apt to occur, the concretion thus escaping into the duodenum by an enlargement of the ostium of the common bile-duct from ulceration or sloughing. Roth, who has paid special attention to this condition, found it five times in twenty-five cases of biliary fistula. These gallstones are usually smaller than those cansing gall-bladder intestinal fistula, are seldom larger than filberts, and do not often cause intestinal trouble. Biliary gastric fistula is less common than might be thought, for the pylorus is not infrequently adherent to the gall-bladder. I have only one case to advance from my own experience, and in that instance the romiting of gall-stones made the diagnosis protable, though the complete recovery of the patient, a woman of fifty, rendered it impossible to be absolutely certsin that the surmise was correct. Murchison was of opinion that all vomited gall-stones must have entered the stomach through a fistula. In one case Jeaffreson"found such a fistala post mortem, a gall-stone having been vomited some time before.

Lutestinal obstruction - Intestinal obstruction from gallstones is such a distinct complication of cholelithiasis, calling for special treatment, that I think it will not be beyond my province to consider it, and as the chief variety of obstraction is necessarily associated with fistula it seems to me convenient to consider it here. So much has been wxitten about obstruction from gall-stones that at first sight it wight seerr to be a somewhat common ailment; such, however, is not the case, as may be gathered from the fact that on inquiring of the registrars and pathologists I found only four such cases had been treated during a period of twelve months in some of the largest hospitals in the kingdom, representing 80,000 in-patients, and several hundred thousand out-patients attended to during the same time. Again, only one case, according to Dr. Brockbank, had sccurred in the Manchester Royal Infirmary between 1883 and 1893, during which time 50,000 in-patients had been treated. There are clearly four classes of obstruction of the intestines depending primarily on gall-stones, though by intestinal obstruction from gall-stones is usually understood the impaction of a large concretion in some part of the intestinal tract producing a mechanical block. The varieties are: 1. That due to local peritonitis in the gallbladder region, leading to paralysis of the bowel. The symptoms may be so severe as to resemble strangulation by a band or acute intussusception. The diagnosis will not, as a rule, be difficult, as the history of the occurrence of previous attacks of spasms, though not of necessity followed by jaundice; the similarity to these of the commencement of the attack in question; the severe and persistent pain, at first localised to the rigbt side of the abdomen; the absence of distension at the commencement, and then the occurrence of distension on the right side only, becoming general later; the lateness of the onset of fæcal vomiting, and only after continued retching; the existence of collapse at an early stage owing to the severity of the pain, which is usually relieved by a morphia injection; the usual absence of visible peristalsis ; and, lastily, the onset of jaundice if the concretions have reached the common duct, afford so much guidance that error will not often occur, especially if the patient be $a$ wowan of middle or old age. These cases will, as a rule, yield to general and medical treatment, and it will only occisionally be necessary to resort to operation during the seizure if the symptoms are not subsiding, though subsequerit surgical treatment may be required. 2. Volvulus of the small intestine, dependent on the violence of the colic caused by an attack of gall-stones, or on the contortions induced by the passage of a large concretion through the wall intestine, is probably uncommon. The following are cbbreviated notes of cne of two cases that have come under my personal observation.

Cast 1.-Acute intestinal obstruction in a woman, aged sixty-eigbt years, pperated on Nov. 12th, 1890, by laparotomy, on the eighth day of the obitruction, a volvulus of the small intestine being discovered and untwisted. The bowels were moved bv enema on the sixteenth day after onset of obstruction and the eighth day after operation, and a large gall-stone three inches in circumference was passed, this being manifestly the cause of the obstruction, and, secondarily, of the volvulus. The patient returned home on the twenty-sixth day, and remained quite well when heard of a year subsequently.

Diagnosis. - In this class of cases (volvulus) a positive diagnosis is, probably for the most part, out of the question, except after the abdomen is opened, as volvulus of the small intestine is an extremely rare event, and we know that a large gall-stone may quietly ulcerate its way into the gut without any preliminary warning, the symptoms only arising when the concretion is passing through the small bowel.

Treatment. - In this class, operation holds out the only hope of success.

The fourth class is characterised by obstruction coming on after the original cause has disappeared and depends on adhesions left by the local peritonitis due to gall-stone attacks, or on narrowing caused by the healing of a tistulous opening through which a large gall-stone has made its way into the intestinal tract. Dr. Brockbank refers to a case in the Transactions of the Pathological Society of London, 1852 , in which there was chronic inflammation and thicken ing of the ileum and cæcum, with destraction of the ileocæcal valve, these being dependent on gall-stones found in the thickened and ulcerated bowel. The bowel was dilated above the obstruction and much contracted below. The patient suffered from chronic diarrhoa for three years, which alternated with attacks of obstruction.

The third class is the most important variety of obstruction dependent on gall-stones, and is the one furnishing, not only the greatest number of cases, but a considerable number of museum specimens. It is dependent on the mechanical obstruction and damage to the bowel produced by the passage of a large concretion through the intestine.

In a paper read before the Royal Medical and Chirurgical Society in 1894 I related notes of cases illustrating this condition. It is astonishing how few unsuccessful cases are reported, yet we know that the mortality of these operations bas been considerable. In making a study of the reported cases, and especially of museum specimens, one cannot help feeling astonished at finding fatal obstruction depending on quite small concretions and the comparatively easy passage of very large gall-stones. Schuller (Strasburg, 1891), in reviewing 139 publisbed cases, found that the subjects were women in 74.3 per cent., and of these 75 per cent. occurred in women over fifty years of age, though instances were found from eighteen to ninety-four years of age. Lobstein (Heidelberg) ${ }^{4}$ gives the most common age as between forty and sixty years. It is a curious fact that although the calculi usually produce intestinal trouble within a few days of reaching the intestine, in some cases they may remain in the bowel for long periods-e.g., in a case under the care of Mr. Eve, ${ }^{5}$ ten years, and in one under the care of $\mathrm{Mr}$. Thomas Smith ${ }^{6}$ probably fifteen years. In Courvoisier's elaborate statistics, out of fifty-three cases examined, be gives the site of obstruction as in 214 per cent. in the duodenum and jejunum; in 65.4 per cent. in the ileum; in 10 per cent. at the ileo-cæcal valve; and in 24 per cent. in the sigmoid flexure. The disease is peculiarly fatal one - of 280 cases collected by Schuller. Dufort, and Courvoisier 156 died, or 52 per cent. Kermisson and Rochard, ${ }^{7}$ of 105 collected cases, gave the mortality as 50 per cent. The cases that recovered lasted on the average eight days, those that died ten days, but the duration of obstruction may vary from one to twenty-eight days. A case reported by Sargent ${ }^{8}$ actually died apparently from the intensity of the pain, after symptoms lasting only half an hour. Lobstein ${ }^{9}$ collected 92 cases; of the 61 not operated on 32 recovered, the remaining 29 died from peritonitis or exhanstion; of the 31 operated on 12 recovered, but as many of the 19 which died were moribund when operated on, their death cannot be charged to operation. As more than one large concretion may be present in the gut at the same time, in a few cases the symptoms of obstruction have been known to recur once, twice, or three times after the first concretion has been parted with. Dr. Maclagan 10 has described two cases of this kind, and Mr. Clutton ${ }^{\text {II }}$ has described another in which be operated successfully within twenty.four hours of the

4 Annals of Surgery, January, 1896

Transactions of the Clinical Society, 1895 . 6 The Lanckr. Dec. 3rd, 1887.

Archives Générales de Médecine, February, 1892 8 Brit. Med. Jour., 1879.

9 Annals of Surgery, Javuary. 1896

10 Transactiuns of the Clinical Suciet $y$, vol, skit, p. 67. 12. Ibid., p. 99 
onset of the second seizure and manipulated the stone through the ileo-crcal valve. The symptoms are those of acnte intestinal obstruction from other causes, with early fæcal vomiting and severe abdominal pain. 'The higher in the gut the impaction the more violent, as a rule, will be the symptoms. The obstruction can only very rarely be felt through the abdominal walls. Although in one of my cases $I$ was able to make a probable diagnosis from the history of previous gall-stone attacks extending over several years, yet in many cases there is absolutely no previous history to guide one, and it is quite impossible to say whether or not the attack is one dependent on the cause in question or on a volvulus, or band, or internal hernia, which, if left, must inevitably lead to death, and that speedily. The age and sex, together with the history of chronic dyspepsia and pain in the hepatic region, are, however, well worth bearing in mind as well as the early and persistent vomiting and visible peristalsis limited to the small intestines.

Treatment.-If the diagnosis could always be made with certainty I think this is a condition in which medical and expectant treatment might be fairly given a trial, since we have ample evidence of large gall-stones having safely passed without other treatment. But we must not forget that 52 per cent. of cases treated on medical and expectant lines are fatal, and although surgery has not yet shown a much greater percentage of recoveries it is because surgical means are frequently only resorted to when the case is hopsless and after all other means have been tried. When it is borne in mind that there are no symptoms peculiar to this form of obstruction, and that the course pursued by an obstruction by a bund or by an internal hernia may be exactly the same as in gall-stone obstruction, the surgeon who waits beyond the period when an operation may be undertaken with every hope of success is incurring a very serious responsibility. With regard to the method of treatment after the abdomen is opened and the cause found, if the gall-stone can be easily crushed through the intestinal coats, without too much force being required, so much the better, but that failing enterotomy should be performed, as it can be done very quickly and with very little damage to the bowel. Should the patient be too ill to bear a search being made for the obstruction, enterotomy, or perhaps short circuiting, might be performed in order to give temporary relief, the cause of the obstruction being afterwards removed, if not passed naturally. As to when operation should be performed, that is part of a general question which each surgeon will have to answer for himself in every individual case, as no definite rule can possibly be formulated which will apply to all cases. The surgeon will, as a rule, not be called in before decided symptoms of intestinal obstruction have manifested themselves and until medical means have been fully tried; in such cases it would seem to me to be idle waste of time to delay surgical intervention until the patient is so exhausted that operation is only undertaken as a dernier ressort, when the subject is almost moribund. If, however, the case be seen at an earlier stage morphia will have to be given to relieve the pain, and I have usually recommended extract of belladonna in quarter-grain doses every four hourz, the stopping of all food by the mouth, and the administration of one or more large syphon enemas, given slowly with the buttocks elevated. If relief does not speedily follow and the diagnosis is not clear, chloroform anæsthesia may assist in two ways. In the first place it enables a thorough examination of the abdomen and at times a diagnosis of the cause to be made; and, secondly, the manipulation, if made methodically, may reduce a hernia or volvulus or may possibly help onwards an obstruction. This failing and the symptoms persisting I should operate, and at this comparatively early stage I feel sure with every prospect of success.

Tumours of the gall-bldader and bile-ducts - If by tumour be understood new growth, then tumours of the gall-bladder and bile-ducts are not common; but if we accept the usual interpretation of the term and include all enlargements as tumours we shall find them by no means rare.

Distension of the yall-bladder.-A tumour of the gallbladder through distension with bile must be $a$ rare phenomenon, though it is sometimes described as an accompaniment of a gall-stone attack where the concretion is impacted in the common duct; even in such a case it mast be a symptom of short duration, since if the impaction be complete, the bile speedily becomes absorbed and gives place to distension by mucus. A perceptible tumour formed by distension with gall-sìones is aiso rare, unless it happens that some have become impacted in the aystic duct, when a gradual enlargement from the retained mucus will follow. have removed as many as 750 gall-stones from a gall-bladder during an operation, and yet that gall-bladder could not he felt as a distinct tumour. Occasionally a large single galir stone may form a hard perceptible swelling below the liver. but such is very rare. Calcified gall-blajder, which is due to cholelithic catarrh, may lead to the formation of a harc. rounded, painless tamour, and this is evidently not very uncommon if we may judge of its frequency by specimens in the museums.

Hydrops and dropsy of the gall-bladder are terms used to denote distension of the gall-bladder by mucus. It may result from any obstruction in the cystic or common ducts, whether due to gall-stones, stricture, or growin in the ducts or to cancer of the head of the pancreas, provided that the gall-bladder has not atrophied as the resuir of previous gall-stone irritation. It is due to the gradnal accumulation of the natural secretion of the mucous lining. and may attain such a size as to be mistaken for an ovarian cyst, as in cases reported by Mr. Lawson Tait and hy Professor Kocher, though it is uncommon to find the tun our of greater size than from 15 to $20 \mathrm{oz}$. capacity. Not only may the cavity be dilated, but its walls may be enormons!y hyper trophied so as to form a distinct tumour. If the obstractinn be associated with inflammation the contents of the galibladder may become puralent and an empyema of the gall bladder result, the symptoms and complications of which have already considered.

signs.-Enlargements of the gall-bladder may vary from a tumour just perceptible to the touch to one of such a size as almost to fill the abdomen, though one of greater sitso than a large pear is exceptional. The same tumour may also vary in size at different times, this variation being frequenty found in gall-stone obstruction. The symptoms of turaour of the gall-bladder depend for the most part on the cause and in consequence vary considerably, at times being sligh and unimportant, at others both urgent and serious. The gall-bladder, as a rule, enlarges downward and forward in a line which, drawn from the ninth or tenth costal cartilage crosses the linea alba a little below the umbilicas, but the position of the tumour varies with the size of the liver. When that organ is of normal size the neck of the ralibladder is opposite the ninth costal cartilage, whereas when the liver is enlarged the gall-bladder will be pushed down so that the neck of the tumour may be opposite to, or even below, the umbilicus. If uncomplicated it will have a smooth rounded, and pear-shaped outline, the larger end being below. quite free, and movable from side to side, the upper en being fixed and passing under the lower margin of the liver at the fissure of the gall.bladder. A distinct sureus between the liver and gall-bladder is nearly always perceptible to the touch if the warmed flat hand be laid ove the right side of the abdomen and the patient be told to taise a deep breath, when the tumour and the liver will descerud together and pass under the fingers. Bimanual palpation will frequently throw additional light on the case, the right hand being placed in front of the abdomen and the left ove the right loin, making gentle pressure forwards. In other cases additional information may be obtained by placing the patient in the genu-pectoral position and passing the flot hands round the abdomen from behind, when a tumow of the gall-bladder will rest distinctly on the hand, and on deep inspiration it can be felt to move just beneath the abdoninal walls, the upper surface of the liver also being in this way capable of palpation. The swelling is, as a rule, far too tense and hard for fluctuation to be elicited, though at times this sign may be obtained. In some of the larger swellings is thrill almost like the hydatid fremitus may be felt on gently flicking the tumour with the finger-nail. Percussion by no means always elicits dulness coextensive with the tumour, especially if the surrounding intestines be distended, so that dulness on percussion is a very variable sign, and palpotion will be found more reliable. Inspection of the abdomen with the patient recumbent will at times show the tumour descending on respiration, but this sign is astually only to be ohserved in thin patients and in cases uncomplicated with inflammation. When there is inflammation and matting of the adjoining viscera a fixed swelling may be seen over the right bypochondrium, with dulness on percussion and malked tenderness. Tenderness on palpation is a variable symptom depending on the presence or absence of local peritonitis, it being, as a rule, absent in uncomplicated enlargementis of the gall-bladder. Jaundice may complicate tumours of the gall-bladder, both being dependent on the same cause-a 
blocking of the common bile-duct. Although not absolutely pathognomonic of malignant disea se, the combination $:$ hould always raise a suspicion of cancer of the head of the pancreas or of the liver or bile-ducts, especially if it be associated with great loss of flesh and strength and with absence of characteristic gall-stone pain. I have observed in a considerable number of cases distension of the gallbladder with jaundice to be associated with malignant disease, but mush less often the combination of tumour, jandice, and gall-stones, the explanation of this apparent anomaly being that the gall-bladder frequently becomes diminished in size and adherent as the result of gall-stone irritation, so that when the common duct becomes blocked by a calculus jaundice occurs without distension of the gallbladder, as it is unable to expand. If, however, the common duct becomes blocked by gall-stones before the gall-bladder has contracted and formed adbesions there may be the combination of jaundice and tumour. If the common duct be blocked by tumour, the gall-bladder, not having been subjected to irritation, and therefore not baving become contracted, will at once distend. Thus in malignant disease of the head of the pancreas we find the usual combination of jaundice with tumour of the gall-bladder. Gall-bladder tumours usually contain mucus, occasionally pus, rarely bile. In all cases when the cystic duct is obstructed and inflammation has not followed, mucus alone is present, though when infl immation co-exists pus or muco-pus may be found. In obstruction of the common duct by gall-stones the gallbladder, though usually contracted, may be found distended by bile at first and mucus later; though, as a rule, the swelling subsides more or less rapidly and no tumour persists, the gall-bladder shrinking. When the obstruction becomes absolute, as in malignant disease of the head of the pancreas, the tumour formed is persistent, and although the block is in the common duct, bile soon ceases to reach the gall-bladder, and the tumour is always found to contain mucus This occurs on account of the backward pressure preventing the excretion of bile, which, though formed by the liver-cells, is Immediately taken up by the lymphatics.

Diagnosis - Tumours of the gall-bladder may have to be diagnosed from :-(1) Movable right kidney; (2) tumour of the right kidney or of the suprarenal capsule; (3) tumour of the intestine or fæcal impaction; (4) tumour of the liver; (5) pyloric tumour ; and (6) abnormal projection of the liver. The diagnosis of enlargement of the gallbladder from movable right kidney is, as a rule, easy in thin persons ; but in those who are stout or have tense or strong muscular abdominal walls difficulties may and do arise, which can, however, usually be overcome by examination under an anæsthetic. In the case of renal tumour as well as in movable kidney, by distending the intestine with gas the kidney will be pressed back into the loin, but the gall-bladder will be pushed up towards the liver and made more prominent. The last test is usually also sufficient to enable a diagnosis to be made between a distended gall-bladder and a tumour of the right suprarenal body; but tbis is not always reliable, as in a case I saw with Mr. Kebbell, of Flaxton, Ziemmsen's test pushed the swelling upwards, and on performing abdominal section a sarcoma of the suprarenal capsule was found and removed.

In tumour of the intestine or of the pylorus the associated stomach or bowel symptoms are usually sufficient to enable a diagnosis to be made, but when in doubt distension of the stomach or bowel with gas will belp to clear it up, or examination under an anæsthetic will afford assistance. Tumour of the liver itself, either cancer or bydatid disease, may be almost indistinguishable from one of the gall-bladder though the presence of nodules in the liver, with the history and other symptoms of malignant disease, will usually be sufficiently distinctive in cancer, while the less localised and more generally fluctuating swelling, together with the longer history and absence of pain, will distinguish bydatid tumour. It should not be forgotten that the right lobe of the liver may have an abnormal projection, either in the site of the gall-bladder or to the right of that position, which may at first be mistaken for an enlarged gall-bladder; but the absence of symptoms, together with careful bimanual palpation, will usually enable a correct diagnosis to be made; and, as Professor Riedel has pointed out, the gall-bladder may frequentiy be felt apart from the swelling or at the top of it. Pancture with an exploring syringe would, of course, give valuable information, but this should not be lightly andertaken, as it is not devoid of risk, death having occurred on more than one occasion as a direct result of this apparently slight operative procedure. If it is decided to employ an exploring needle the aspirator should always be used, in order that the tense cyst may be completely emptied, otherwise leakage from the puncture is almost certain to occur. After the abdomen has been opened I have seen a puncture of the tumour by a small exploratory syringe to pour out fluid in a forcible stream, showing what would have occurred had the purcture been made tbrough an unopened abdomen. In case of doubt, especially where the symptoms demand interference, exploration of the tumour through a small abdominal incision can be undertaken with very little risk and at the same time further treatment where called for can be carried out. Of the tumours dependent on new growth, cancer of the gall-bladder is the most important, innocent growth, except of inflammatory origin, being extremely rare, unless it be true, as Zenker suggests, that an adenoma first develops in the gall-bladder, and subsequently becames transformed into adeno-carcinoma. Dr. Rolleston ${ }^{12}$ reports a case in which this sequence apparently occurred in the bile-duct of a woman from whom a papilloma was removed, the growth being in immediate contact with a gall. stone. After some months she returned with a growth in the same region, presumably malignant. Cancer of the gallbladder is by no means frequent, and as a primary affection is somewhat rare. Musser collected all the reported cases in the Boston Medical and Surgical Journal of Dec. 15th, 1889, and Dr. Rolleston published an extremely interesting paper on the subject in the Medical Chronicle of January, 1896. It is usually secondary to gall-stones or to cancer of adjoining organs, and in the latter case is not amenable to surgical treatment. The very frequent association of cancer of the gall-bladder with gall-stones is an undoubted fact, and in all probability there is a connexion between the two diseases. Zeurkert ${ }^{15}$ found gall-stones in 85 per cent. of cancers of the gallbladder, and Mnsser, from an analysis of 100 cases, gives the proportion associated with gall-stones as 69 per cent., which may, however, be an under-estimate, as it is well known that gall.stones may produce serious irritation and then pass into the alimentary canal, so that their effects may remain, although the cause may not be discovered. According to Schroeder, 14 per cent. of all cases of gall-stone patients suffer at some time from cancer of the biliary passages, and Naunyn is of opinion that half the cases of chronic jaundice diagnosed as cholelithiasis are com. plicated with cancer or are due to cancer alone. This latter statement is of extreme importance, since operation in the presence of cancer and chronic jaundice is very fatal. The two theories which have been current to explain the co-existence of gall-stone with cancer of the liver are, first, the "irritation" theory, that gall-stones are formed first, and by acting as foreign bodies set up irritation which leads to malignant growth; and second, the "concentration" theory. that gall-stones arise as a secondary result from stagnation of bile in the ducts caused by their obstruction from malignant growth. Mr. C. Beadles, ${ }^{1 *}$ in a paper read before the Pathological Society of London, stated that out of 100 post-morlem examinations at the Cancer Hospital 4 were cases of primary carcinoma of the liver, and all had calculi in the gail-bladder; 36 had secondary carcinomatous growths in the liver, but there were no gall-stones present in any of them. Of 9 cases of primary carcinoma of the bladder a Colney Hatch Asylum 5 were males and 4 females, and gall-stones were present in 7 , being absent in 1 male and 1 female. These facts support the theory of irritation. Simple growths in the gall-bladder are not of great clinical importance except as precursors of malignant disease.

symptoms and signs. - If the growth be primary there will be the history of a more or Fess rapidly growing tumour developing under the right costal margin, accompanied at first by a sense of discomfort, shortly changing to pain, which is often worse at night, and which, though at first localised to the right bypochondrium and epigastrium, usual]y, before great advance has been made, extends round the side to the rigbt infra-scapular region. When the enlargement is first noticed it is felt as an egg-shaped swelling beneath the liver, descending on aspiration. The tumour is hard to the touch and very slightly or not at all tender to pressure. At a later stage it becomes more fixed and more diffused, and nodules may develop and be felt on its superficial surface. As the growth

12 Transactions of the Royal Medical and Chirurgical Society,

January, 1896.
13 Deutsche Archiy für Klinische Nedicinische, 1889.
14 THE LANCET, March 9th, 1895. 
extends it invades the liver and sometimes the duodenum and stomach. Dissemination is rare. According to the invasion or not of the hepatic or common bile-ducts, so will be the presence or absence of jaundice, but in nearly half of the cases some degree of icterus will be found as the disease adrances owing to the presence of catarrh of the bile-ducts. Interference with the action of the bowels, even to partial or complete obstruction, at times occurs. General failure of health, continued wasting, with loss of strength, ascites, and marked cachexia, characterise the later stages. Perforation may occur and hasten the end by the onset of general peritonitis. If gall-stones be present there will be the usual antecedent history of cholelithiasis. Where gallstones with jaundice complicate cancer of the gall-bladder exacerbations of pain will usually be accompanied by rigors and fever, "ague-like attacks" with an intensification of the icterus, and in such cases petechiæ in the skin with hæmorrhage from the nose and rectum usually supervene.

Dixgnosis.-Cancer of the gall-bladder may usually be diagnosed by the progressive character of the disease and by the presence of the characteristic hard tumour; but it is by no means always easy to diagnose cancer from a tumour formed by matted intestines due to local peritonitis in the neighbourhood of the gall-bladder. When in doubt exploration is probably the best method of settling it, as at the same time treatment may be carried out.

Treatment.-The alleviation of symptoms, especially of pain, by sedatives is practically all that can be done except in those rare cases where the disease is limited to the gallbladder, when cholecystectomy may be performed. In only one case out of several on which I have operated have I found the disease sufficiently limited to permit of excision, ana even in that instance I had to perform partial hepatectomy. ${ }^{15}$

Tumours of bile-ducts. - Tumours of the bile-ducts, per se, only occasionally form a projection so large as to be distinguished through the abdominal walls. Tumour, however, in such cases is as a rule present sooner or later on account of the obstruction in the ducts and secondary distension of the gall-bladder. The common duct has been found dilated to such a size as to form a cystic tumour presenting all the characteristics of a distended gallbladder, the gall-bladder itself bing atrophied. In two cases of this kind I performed, in the one choledochostomy after cholelithotrity, the patient making an excellent recovery ; in the other choledochenterostomy after cholecystectomy, the patient also doing well. Although hitherto the results of choledochostomy have not always been favourable, probably in consequence of the fact that extreme distension of the bile-duct is often accompanied by infection of the biliary passages, it would be well to reserve our opinion as to the prospects of the operation until we have more experience of it. Very little information can as yet be obtained on this subject, cases of distension of the common bile-duct being very rare, and those in which surgery has intervened still more exceptional. An interesting case is reported by Mr. W. P. Swain in THE LANCET of March 23rd, 1895, in which he connected a dilated bile-duct to the jejunum by one of Murphy's buttons. The size of the tumour, which occurred in a girl aged seventeen years, and which was associated with gall-stones, may be gathered from the fact that over seven pints of fluid were withdrawn from it at the time of operation. Three months after the patient was progressing satisfactorily except for an occasional rise of temperature, and for the fact that the button had not been passed.

The new growths found in the bile ducts may be simple or malignant. of the malignant we must take into consideration the two classes, primary and secondary, the former arising most frequently as the result of gall-stone irritation, the latter by extension from neighbouring organs. Papilloma is probably an earlier stage of cancer and is rare. These growths are usually found between the ages of fifty and sixty years, and, unlike cancer of the gall-bladder, where 75 per cent. of cases occurred among women, the disease attacks both sexes equally. If forming in the cystic duct jaundice will be absent at first, only coming on when the growth advances so far as to pass into the common duct and obstruct the passage of the bile, or if, as is not uncommon, catarrh of the bile ducts supervenes. The gall-bladder enlarges at an early

15 Transactions of the Royal Medical and Chirurgical Society, rol. Ixsix, stage, and this will probably be the earliest sign; pain may be absent unless gall-stones exist, when the usual spasmodic pains will occur so long as the muscular coat of the gallbladder retains its contractile power. When the growth is in the common duct jaundice comes on at an early stage and persists throughout, the liver gradually increasing in size and the gall-bladder also enlarging ultimately. Supparative cholangitis is apt to supervene, the case then taking on a more acute course and being accompanied by fever, ague-like attacks, and rapid loss of flesh and strength. Needless to say, the disease is uniformly fatal, though operation mav delay the final catastrophe. Cancer of the ampulla of Vater has received some attention from M. Hanot, ${ }^{16}$ M. Durand-Fardel, ${ }^{17}$ M. Rendu, and Dr. Rolleston. ${ }^{18}$ Two forms at least are described, one arising from the intestinal walls of the ampulla and the other from the orifice of the common bile duct and the pancreatic duct. They are characterised by intermittent jaundice, wasting, and cachexia, but as a rule pain is absent. It was probably first described by MacNeal ${ }^{19}$ in 1835, and later by Stokes ${ }^{20}$ in 1846

Diaynosis. - The diagnosis of new growth in the bile-ducts from gall-stones is practically impossible, as the symptoms are the same, and in fact the two frequently co-exist. The abserce of pain in some cases and the rapid deterioration of health may afford a little help, but in some cases the pain may be as acute as in cholelithiasis. Cystic dilatations of the bile ducts are of ten indistinguishable from enlargements of the gall-bladder, for which they are usually mistaken; but they may resemble a cyst of the pancreas or a bydatid tumour of the liver. As, however, the treatment in all these conditions is abdominal section, no harm will be done if the diagnosis is only completed when the abdomen is opened.

Treatment.-The operative treatment of these tumours is in its infancy and bas thus far not proved uniformly satis factory. Any growth should be removed if possible, but where that is impracticable the dilated gall.bladder or ducts may be opened and drained, or, better still, drained into the duodenum or jejunum by means of one of Murphy's buttons. If the cause be a removable one, such as a gall-stone, it should be taken away. Choledochostomy has not yielded good results in cystic dilatation of the bile.ducts, my own case being, I believe, the only example of recovery after the operation, whereas the experience of performing an anastomosis between the cyst and the intestine, though as yet slight, has been so satisfactory as to establish its claim to being considered the best method of treatment.

15 Archives Générales de Médecine, November, 1896. 7 La Presse Médicale, 1896.

18 Transactions of the Royal Medical and Chirurgical Society, 1895. 19 North American Archives, Baltimore.

20 Dublin Quarterly Journal of Medical Science.

CRIME IN WILTshiRE.-The report issued by the chief constable of Wiltshire and presented at the meet. ing of the standing joint committee last week showed that 727 persons had come under the cognisance of the police during the past quarter, of whom 26 , were committed for trial, 495 summarily convicted, 71 quasi-criminal cases, and 135 discharged. There had been an increase of 36 in the numbers of persons proceeded against.

University of CAMbridge. - The ViceChancellor for the next academical year will be a Doctor of Medicine, Dr. A Hill, Master of Downing College, and Lecturer in Advanced Human Anatomy, having been elected to the office on June 1st. The Deputy Professor of Pathology, Dr. A. A. Kanthack, announces courses in Pathology, Bacteriology, and Morbid Anatomy and Histology, during the ensuing long vacation, beginning on July 7th and 8th. Some of these will be adapted to the requirements of candidates for the Diploma in Public Health. It is proposed to confer honorary degrees on the Archbishop of Canterbury, the Marquess of Lansdowne, Lord Russell of Killowen, Chief Justice Way, a number of the colonial premiers now in England, Sir G T. D. Goldie, Sir John Kirk, Sir Arthur Arnold, and Sir W. H White. On May 27th the following medical and surgical degrees were conferred:-M.D.: H. Walsham, M.A. Cains, and N. L. Hood, M. A., M.B. and B.C. Downing: L. T. R. Hutchinson, B A. Trinity, W. McDougall, B.A. St. Jobn's, L. T. Giles, B.A. St. Peter's, R. D. Parker, BA. Caius, and W. B. Heywood, B.A. Emmanuel. M B oniy: W. M. sing, M.A. Christ's. 